\title{
Annals of General Hospital Psychiatry
}

\section{Brain mechanisms of emotion: initial MEG findings}

\section{A Papanicolaou*}

\author{
Address: Professor and Director, Division of Clinical Neurosciences, Department of Neurosurgery, University of Texas - Huston Medical School, \\ USA \\ * Corresponding author
}

from International Society on Brain and Behaviour: Ist International Congress on Brain and Behaviour

Hyatt Regency Hotel, Thessaloniki, Greece, 20-23 November, 2003

Published: 23 December 2003

Received: I November 2003

Annals of General Hospital Psychiatry 2003, 2(Suppl I):S29

This article is available from: http://www.general-hospital-psychiatry.com/content/2/SI/S29

In a series of two experiments we sought to identify spatio-temporal brain activation profiles representing the neuro-physiological mechanisms generating affective experience. Magnetoencephalography (MEG) was used to acquire these profiles in 12 healthy participants while they viewed pictures rated as affectively neutral, and pictures rated as affectively positive and affectively negative. Consistent activation of the occipital and posterior basal temporal cortices was observed in each subject in both experiments. This pattern of activation occurred immediately after stimulus presentation, lasted approximately $270 \mathrm{~ms}$. and was common to all stimuli, both affective and affectively neutral. It is, therefore, not affect-specific but it represents the brain mechanisms of visual recognition. A second pattern of equally consistent activation followed the first. This pattern was obtained during the negative affect condition only. It involved activation of the left mesial temporal cortex and was observed in every subject during both experiments. In addition, during the second experiment, this pattern included consistent activation of the left cingulate gurus. These results constitute the first demonstration of the efficacy of functional neuroimaging to identify structural components of the brain mechanisms of affective experiences in individual subjects, without the use of group statistics. They also constitute the first demonstration of the capacity of MEG to identify distinct brain mechanisms of recognition versus affect production with millisecond resolution. 